\title{
The El Niño events: their classification and scale-invariance laws
}

\author{
Adriano Mazzarella and Andrea Giuliacci \\ Osservatorio Meteorologico, Dipartimento di Scienze della Terra, \\ Università degli studi «Federico II», Napoli, Italy
}

\begin{abstract}
Many point indices have been developed to describe the El Niño/Southern Oscillation but the Multivariate ENSO Index (MEI) is considered the most representative since it links different meteorological parameters measured over the tropical Pacific. The monthly values of the positive feature of MEI (interval: 1950-2008) have been ranked as a catalogue of El Niño events according to a semi-quantitative strength index ranging from 1 to 6 . Such a catalogue is shown to be scale invariant in respect both to strength index and to times of occurrence, suggesting that the ocean-atmosphere interaction phenomenon at the basis of El Niño belongs to the class of dynamical processes which are in a self-organized critical state.
\end{abstract}

Key words fractal geometry - scale-invariance MEI, El Niño, SOC

\section{Introduction}

The El Niño Southern Oscillation (ENSO) is a complex atmospheric and oceanographic phenomenon that has been the object of much attention in recent years. Besides their scientific importance as major climatic modifiers, warm ENSO events (i.e. El Niño) have deep economic and social consequences. The 198283 El Niño motivated a rush of research activity attempting a better understanding of the phenomenon and, hopefully, forecasting its surging and duration. With this purpose, many mathematical models have been developed to describe ENSO but the predictability of El Niño

Mailing address: Dr. Adriano Mazzarella, Osservatorio Meteorologico, Dipartimento di Scienze della Terra, Università degli studi «Federico II», Largo S. Marcellino 10, 80138 Napoli, Italy; e-mail: adriano.mazzarella@unina.it events is still an open question. The monitoring of ENSO is mostly based on observations of sea surface temperatures (averaged to single values over large regions within the equatorial Pacific Ocean identified as Niño 4, Niño 3 and even Niño 3.4) and on differences between Tahiti and Darwin atmospheric pressure in the whole tropical Pacific Basin (Rasmusson and Carpenter, 1982; Ropelewski and Jones, 1987; Allan et al., 1991; Trenberth, 1997; Können et al., 1998; Wallace, 1998). Such point measures are useful indicators of the ENSO phenomenon but not representative of the coupled ocean-atmosphere phenomenon: the complexity of the climate system cannot be understood on the basis of simple and point measures. The NOAA's Climate Diagnostics Center in Boulder Colorado has developed a more holistic approach regarding a Multivariate Enso Index (MEI) (Wolter and Timlin 1993; 1998) whose positive feature represents the warm ENSO phase related to El Niño occurrence. Recently, Mazzarella et al. (2009) identified a 60-month cycle in MEI series whose maxima and minima values are found to be in correspondence of El Niño and La Niña occurrences. Here we visit the month- 
ly series of MEI as a sequence of El Niño events according to robust scale-invariant statistical analyses.

\section{Data collection}

The data consist of the monthly values of Multivariate Enso Index (MEI) (interval: 19502008) as computed by Wolter and Timlin (1993; 1998), taken from website:

http://www.cdc.noaa.gov/people/klaus.wolter/ MEI/table.html,

where each monthly value is centered between the preceding and subsequent month: for example, the January value represents the value centered between the December-January months and so on. The MEI is a multivariate measure of the ENSO signal derived from six main observed variables over tropical Pacific and belonging to Comprehensive Ocean-Atmosphere Data Set (COADS): sea-level pressure, zonal and meridional components of the surface wind, sea surface temperature, surface air temperature and cloudiness of the sky. The COADS data set is available at a sufficient resolution such that large scale patterns (as opposed to point measurements) can be used in relation to each other to obtain a more comprehensive measure of ENSO-related interactions. The MEI values are standardized with respect to a 1950-93 reference period and are computed as the first unrotated Principal Component (Miranda et al., 2008) of all six combined observed fields with a mean equal to zero and a standard deviation equal to 1. Such values are expressed as percentages of standard deviation (Wolter and Trimlin 1993; 1998) and are reported on fig 1.

\section{El Niño events and their cumulative distribution}

To analyse the MEI monthly series as a catalogue of El Niño events, we have computed many preliminary cumulative-distributions of the positive monthly values of MEI according to different grouping intervals to account for the error due to the estimating. The results of the different trials have shown that the interval of

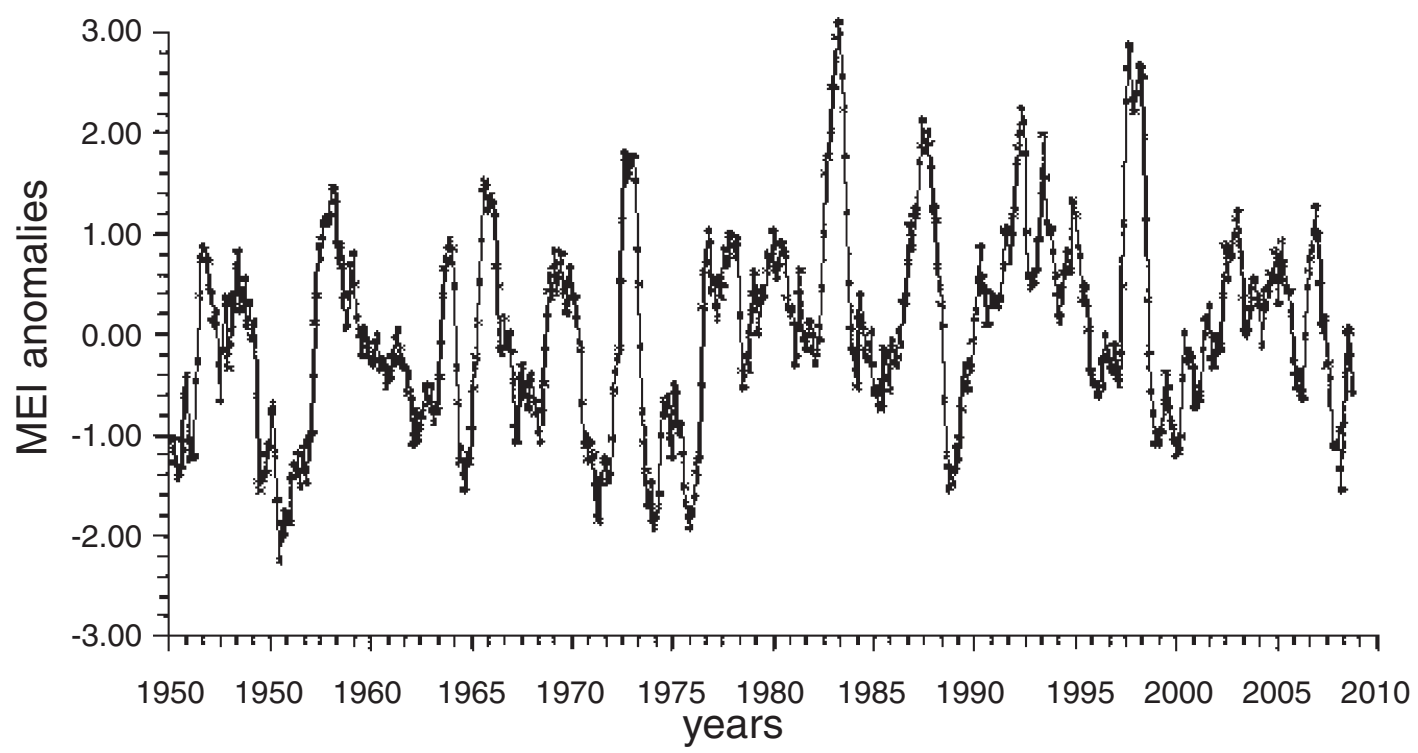

Fig. 1. Time series plot of MEI monthly values from January 1950 to December 2008. 


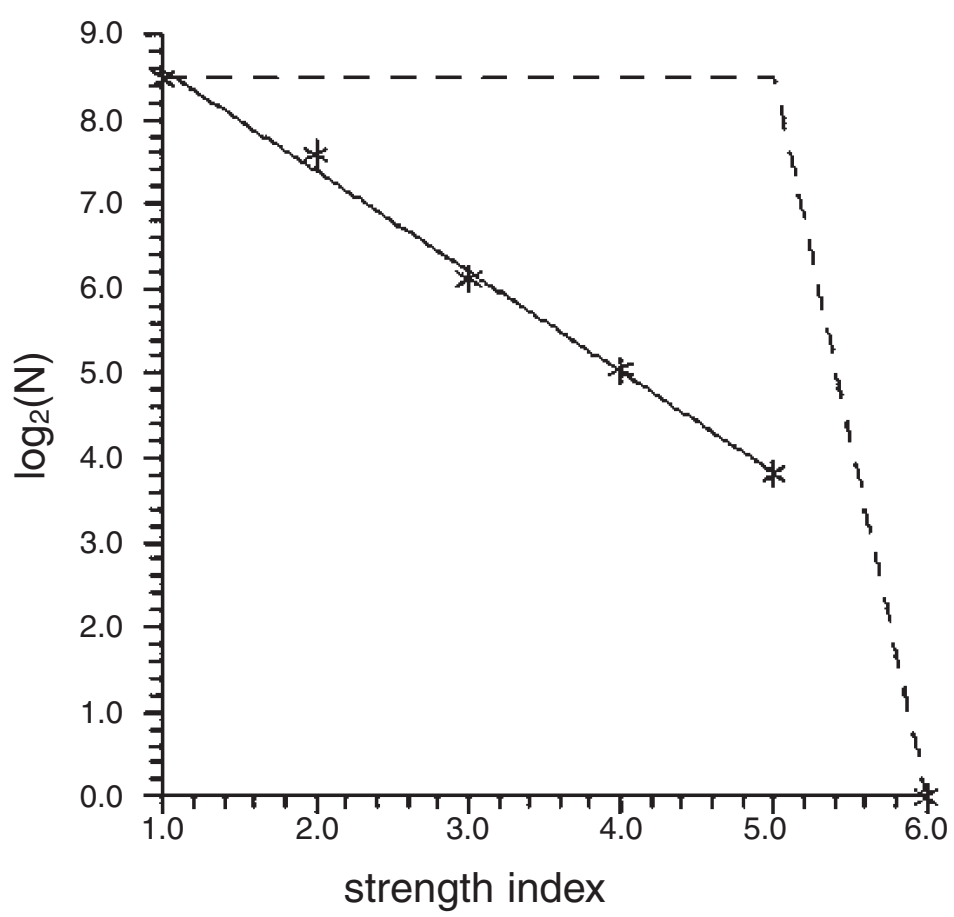

Fig. 2. Cumulative distribution of the logarithm of the number of El Niño events since 1950 versus their strength index $(S I)$. The dashed line represents the relative random distribution.

0.6 unit yields the best-constrained shape of the cumulative distribution (fig. 2). This has ranked the MEI positive monthly values inside a catalogue of El Niño events according to the following semi-quantitative strength index (SI) ranging from 1 to 6 : very weak $(\mathrm{SI}=1)$ if $0 \leq$ $\mathrm{MEI}<0.6$, weak $(\mathrm{SI}=2)$ if $0.6 \leq \mathrm{MEI}<1.2$, moderate $(\mathrm{SI}=3)$ if $1.2 \leq \mathrm{MEI}<1.8$, strong $(\mathrm{SI}=$ 4) if $1.8 \leq \mathrm{MEI}<2.4$, very strong $(\mathrm{SI}=5)$ if 2.4 $\leq \mathrm{MEI}<3.0$ and extreme $(\mathrm{SI}=6)$ if MEI 3.0. The so constructed catalogue is found to contain a number $N$ of El Niño events equal to 356 whose logarithmic cumulative distribution is related to SI through the best fitting:

$$
\log _{2}(N)=\mathrm{a}-\mathrm{b} \text { SI }
$$

within the range $1 \leq \mathrm{SI} \leq 5$ and obtained at a level of confidence higher than $99 \%$. Here, $a=$ $6.48 \pm 0.05$ is the $\log _{2}$ of the number of events with $\mathrm{SI} \geq 0$ and $b=1.19 \pm 0.05$ is a measure re- lated to of El Niño generating process. The regression line (3.1) regards a number of pairs $N C$ $=5$ for which the relative confidence level is here based on the null hypothesis of zero correlation that is rejected at the $99 \%$ confidence when the following relationship:

$$
[r \sqrt{(N C-2)} / \sqrt{(1-r)}]
$$

gives a value greater than that provided by the Student distribution for a number of degrees of freedom equal to $N C-2$ and a correlation coefficient equal to $r$ (Mazzarella, 1998). The cumulative distribution of El Niño events, verified to be significantly different from the simulation obtained when the same number of events is randomly distributed, can be here interpreted in terms of scale-invariance of El Niño catalogue if we consider SI as a simple 1-to-6 index of increasing factor of around 2 (fig. 2). It appears that the El Niño catalogue is significantly scale 
invariant and presumably complete, during the period 1950-2008, for all the analysed events with the exception of the event of March 1983, classified as $\mathrm{SI}=6$, i.e., extreme. This indicates that no El Niño event presumably has in the past escaped detection and that the event with $\mathrm{SI}=6$ probably is not representative of the $\mathrm{El}$ Niño process within the 1950-2008 interval.

\section{The Cantor dust model and the time distribution of EI Niño events}

The scale-invariant properties of time distribution of El Niño events can be investigated on the basis of the Cantor dust model that is a boxcounting algorithm (Mandelbrot, 1983; Turcotte, 1997) already utilized successfully in different processes involving seismic (Smalley $\mathrm{Jr}$ et al., 1987), volcanic (Dubois and Cheminee, 1991), and flooding (Turcotte and Green, 1993; Mazzarella, 1998; Mazzarella and Rapetti, 2004) events. To apply the Cantor dust method to a catalogue, we divide the time interval $t_{0}$, over which the series of $N$ events occurs, into a series of $\mathrm{n}$ smaller intervals of length $t=t_{0} / n$ with $n=2,3,4 \ldots$ and compute the fraction $R=$ $N / n$ of intervals of length $t$ occupied by events. If the distribution of events has a fractal structure then:

$$
R=C t^{(1-D)}
$$

where $C$ is a constant and $D$ is the fractal dimension or, equivalently

$$
\log (R)=\log (C)+(1-D) \log (t)
$$

on a log-log scaled plane. The power law (4.2) must be, moreover, significantly different from a distribution in which the same number $N$ of events is randomly spaced in time. It is easy to show that a regular time distribution of events causes an increase in $D$ up to the limiting value $D=1$ that is the dimension of a line. On the other hand, a concentration of events at a definite time corresponds to the limiting value $D=0$ that is the dimension of a single point. As a mathematical representation, the power law (4.2) could be valid over an infinite range.
However, for physical applications, there will be upper and lower limits on the applicability of the fractal distribution. The fractal dimension $(D=1-s)$ is estimated from the slope s that provides the best least square fit of $\log (R)$ on $\log (t)$ at a confidence level higher than $99 \%$ according to relationship (3.2).

\section{Results}

The cumulative distribution of the available 356 El Niño events can be explained in terms of scale-invariance assuming that SI is a simple 1to-6 index increasing with factor of 2 (fig. 2). It appears that the number $N$ of events is linearly related to SI, on a log-log scaled plane, within the range $1 \leq \mathrm{SI} \leq 5$, with a level of confidence higher than $99 \%$ and significantly different from the relative random simulation. This shows that the catalogue is reasonably complete to indicate that in the past no El Niño event escaped detection and that the event with SI $=6$ is not representative of the El Niño process within the 1950-2008 interval. To verify the scaleinvariant features in the recurrence intervals of El Niño events, we have applied the Cantor dust method to their times of occurrence. The smallest time interval chosen was 1 month, gradually increased by a factor of 2 . The fraction $R$ (the fraction of time intervals including an event) as a function of the interval size $t$, on a $\log -\log$ scaled plane, is reported in fig. 3. It shows a linear relationship (significantly different from the relative random simulation and confident at a level higher than 99\%) with a fractal dimension $D$ equal to $0.78 \pm 0.01$ and with a scaling region enclosed between 2 and 32 months. $R$ was found to be not significantly different from the correspondent random simulation (no clustering) for values of $t>32$ months, where the events fill a line $(D=1)$ because biased by 60 month harmonic cycle identified in MEI monthly data (Mazzarella et al., 2009). In fact, a Cantor dust algorithm applied to values of increasing intertimes is found to provide a fractal dimension $D=1$ as soon as the scaling region reaches the value of the period of the long-time modulating cycle. Again, the 60-month cycle is found to bias the relative low-frequency spec- 


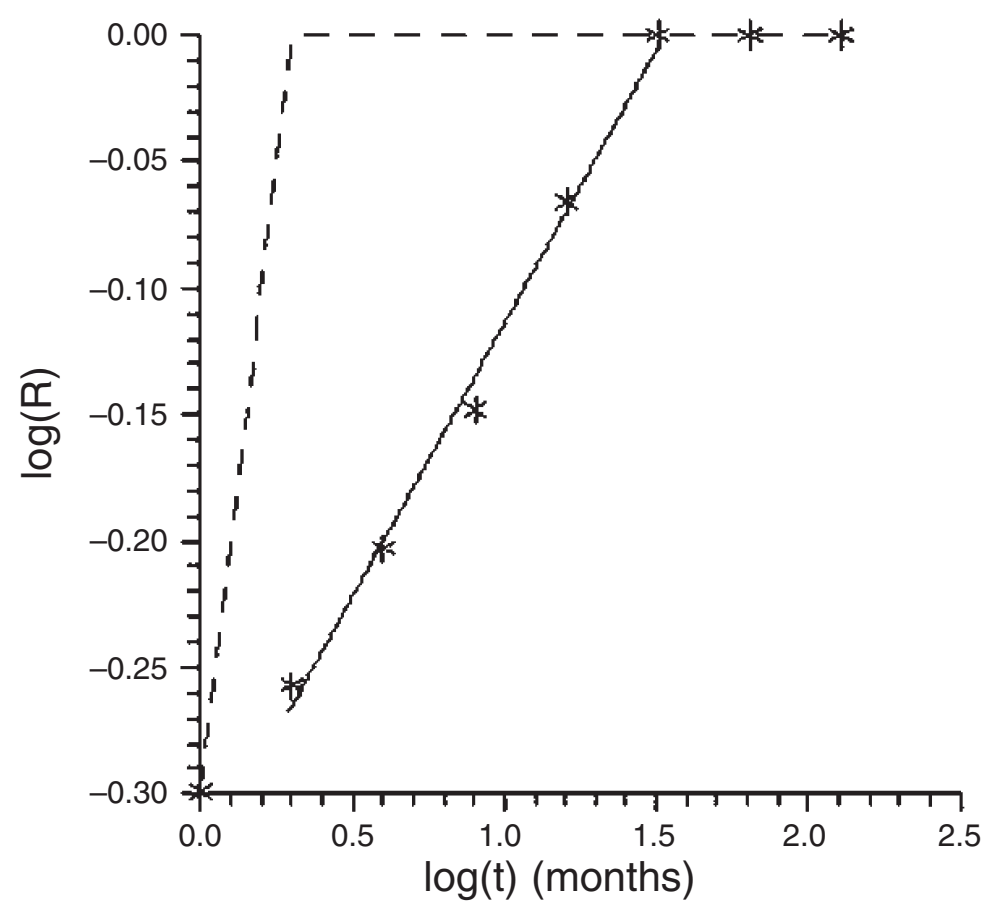

Fig. 3. The logarithm of the fraction $R$ of time intervals of length (in months) including at least one event with $S I \geq 1$ as a function $\log (t)$. The dashed line represents the relative random distribution.

tral estimates of the monthly MEI series available only from 1950 for which the comparison between the fractal dimensions computed utilizing the MEI spectral analysis and the Cantor dust method is not possible.

\section{Discussion and conclusions}

The monthly values of the positive feature of MEI (interval: 1950-2008) have been ranked as a catalogue of El Niño events according to a semi-quantitative strength index SI ranging from 1 to 6 and increasing by a factor of 2 . Such an index corresponds to a qualitative classification of El Niño events as very weak, weak, moderate, strong, very strong, extreme. All the available El Niño events, qualitatively classified as very weak, are shown to be scale invariant in respect to SI, with the exception of the event of March 1983, classified as SI $=6$, i.e., extreme, to indicate that no small event presumably has in the past escaped detection and that the event with SI $=6$ probably is not representative of the El Niño process within the 19502008 interval. The application of the Cantor dust method to the occurrence-times of same El Niño events has identified different patterns of time clustering: a region of no clustering with a fractal dimension $D=1$ (that is the topological dimension of a line) for $t>32$ months and a region of moderate clustering with $D=0.78$ (with a topological dimension nearer to that of line than that of a point) for $2<t<32$ months. The existence of scale-invariant properties in the sequence of El Niño events both with respect to strength and to time occurrence suggests that scale shifts, from small to large scales, are possible, even within a limited range, which might allow a better understanding of the process at the basis of the phenomenon and possibly to assist in the El Niño prediction. On the other 
hand, such invariant-scale properties can be reasonably interpreted as manifestations of the self-organized critical state (Bak and Chen, 1989; Bak, 1996) at basis of El Niño process (Andrade et al., 1995) that depends on numerous meteorological and oceanic factors, linked together in a non-linear way and with numerous feedback processes. It is worth noting that the values of $b$ and $D$ in relationships (3.1) and (4.1), within their scaling regions of validity, represent the values of clustering in strength and in time of El Niño events starting only from 1950. The unavailability of longer series precludes the investigation of their time changes to obtain a better understanding of the phenomenon. For this, the results here obtained should be considered preliminary.

\section{REFERENCES}

Allan, R.J., N. Nicholls, P.D. Jones and I.J. ButterWORTH (1991): A further extension of the Tahiti Darwin SOI, early SOI results and Darwin pressure, J. Climate, 4, 743-749.

Andrade Jr., J.S., I. Wainer, J. Mendes Filho and J.E. MoreIRA (1995): Self-organized criticality in the El Niño Southern Oscillation, Physica A, 215, 331-338.

BAK, P. and K. CHEN (1989): The physics of fractals, Physica $D, 38,5-12$.

BAK, P. (1996): How the Nature works: the science of selforganized critical state, (Copernicus Springer-Verlag, New York), p. 212.

Dubois, J., and J.L. ChemineE (1991): Fractal analysis of eruptive activity of some basaltic volcanoes, J. Volcan. Geoth. Res., 45, 197-208.

KönNen, G.P., P.D. Jones, M.H. KaLtofen and R.J. Allan (1998): Pre-1866 extensions of the Southern Oscillation Index using early Indonesian and Tahitian meteorological readings, J. Climate, 11, 2325-2339.

Mandelbrot, B.B. (1983): The Fractal Geometry of Nature, (Freeman and Company, New York), p. 468.
Mazzarella, A. (1998): The time clustering of floodings in Venice and the Cantor dust method, Theor. Appl. Climatol., 59, 147-150.

MAZZARELLA, A and F. RAPETTI (2004): Scale invariance in the recurrence interval of extreme floods: an application to the Upper Po River valley (Northern Italy), $J$. Hydrol., 288, 264-271.

Mazzarella, A., A. Giuliacci and I. LiRitZis (2009): On the 60-month cycle of multivariate ENSO index, Theor. Appl. Climatol., doi: 10.1007/s00704-009-0159-0.

Miranda, A.A., Y.A. Le Borgne and G. BontemPi (2008): New Routes from Minimal Approximation Error to Principal Components, Neural Proc. Lett., 27, 197-207.

RASMusson, E.G. and T.H. CARPENTER (1982): Variations in tropical sea surface temperature and surface wind fields associated with the Southern Oscillation/El Niño, Mon. Wea. Rev., 110, 354-384.

RoPELEWSKI, C.F. and P.D. JONES (1987): An extension of the Tahiti-Darwin Southern Oscillation Index, Mon. Wea. Rev., 115, 2161-2165.

Smalley Jr, R.F., J.L. Chatelain, D.L. Turcotte and R. PREVOT (1987): A fractal approach to the clustering of earthquakes applications to the seismicity of the New Hebrides, Bull. Seismol. Soc. A., 77, 1368-1381.

Trenberth, K.E. (1997): The definition of El Niño, Bull. Am. Met. Soc., 78, 2771-2777.

Turcotte, D.L. and L. Greene (1993): A scale-invariant approach to flood-frequency analysis, Stochastic $\mathrm{Hy}$ drol. Hydraul, 7, 33-40.

TurCotTe, D.L. (1997): Fractals and Chaos in Geology and Geophysics, (Cambridge Univ. Press,), p. 398.

WALLACE, J.M.(1998): On the structure and evolution of ENSO-related climate variability in the tropical Pacific: Lessons from TOGA, J. Geoph. Res., 103, 14,24114,259 .

WolteR, K. and M.S. TIMLIN (1993): Monitoring ENSO in COADS with a seasonally adjusted principal component index, Proc. of the 17th Climate Diagnostics Workshop, (Norman, OK, NOAA/N MC/CAC, NSSL, Oklahoma Clim. Survey, CIMMS and the School of Meteor., Univ. of Oklahoma), pp. 52-57.

Wolter, K. and M.S. TimLin (1998): Measuring the strength of ENSO events - how does 1997/98 rank?, Weather, 53, 315-324.

(received, May 3, 2009; accepted, September 3, 2009) 